\title{
Difficultintubationapp.com - A difficult airway electronic record
}

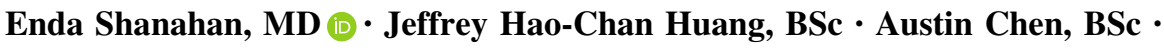 \\ Aftab Narsimhan, BASc $\cdot$ Raymond Tang, MD
}

Received: 13 June 2016/Revised: 19 July 2016/Accepted: 9 August 2016/Published online: 18 August 2016

(c) Canadian Anesthesiologists' Society 2016

\section{To the Editor,}

Inability to secure the airway during general anesthesia can have devastating consequences for the patient. Measures improving the care of patients with a known difficult airway would be beneficial. One area that offers room for improvement is documentation and communication of the difficult airway among healthcare providers. As Benumof tells us "...the patient's responsible doctor must guarantee communication of this difficult airway experience to future care takers so that the near death experience does not become a future death."

Current recommendations from the Difficult Airway Society ${ }^{1}$ and the American Society of Anesthesiologists ${ }^{2}$ include documenting the findings in anesthetic and medical records, enter the patient in an in-hospital and national database/alert system, inform the family doctor, verbally communicate the problem to the patient, and issue them a letter describing the difficulty encountered, instructing them to present this letter at the time of their next anesthetic experience.

Unfortunately, at least $20 \%$ of patients do not recall receiving a letter, and $50 \%$ do not recall the postoperative conversation. ${ }^{3}$ Some institutions do not have a standardized letter containing the pertinent details of the event to issue to patients. Old anesthetic records are also not always readily available at the time patients are seen

E. Shanahan, MD $(\bowtie) \cdot R$. Tang, MD

Department of Anesthesiology \& Perioperative Care, Vancouver

General Hospital, Vancouver, BC, Canada

e-mail: endashan@gmail.com

J. H.-C. Huang, BSc $\cdot$ A. Chen, BSc $\cdot$ A. Narsimhan, BASc Department of Computer Science, University of British Columbia, Vancouver, BC, Canada preoperatively, particularly when treated at different institutions. In addition, a physical letter may be easily lost, or the patient may not remember to bring the letter to his/her appointment. However, the ubiquity of smartphones in our patient population offers an opportunity for clear, accurate, and highly portable communication. To address this communication gap, we developed a website and accompanying smartphone application (Android version currently available; iOS version due for completion in July 2016). The process for its use as follows.

1. The anesthesiologist goes to the website (www. difficultintubationapp.com) and selects the 'create patient letter' option.

2. The anesthesiologist enters the patient's airway management details as prompted and, when finished, generates a hard copy, printable patient letter. The patient is counselled about their difficult airway and given this letter. A copy may be placed in the patient's medical record.

3. The patient's letter includes a URL where the smartphone application can be downloaded and a QR code that contains the information in the letter.

4. When downloading the app, the patients agree to a disclaimer that they own these data and are responsible for its dissemination.

5. The patient can then scan the $\mathrm{QR}$ code and its information into the app.

6. On the next occasion of a general anesthetic or preoperative consultation the patient opens the app and the anesthesiologist can read the information (Figure). The patient also has the ability to export their data as a text file (.txt) which could be emailed and printed or saved on the patients' personal computer, a hard disk, or a cloud storage facility. 
Figure Screen capture of information displayed on the difficultintubationapp.com app

\section{Difficult Intubation Difficult Intubation}

Difficult Intubation Information

Date : 6/17/2016

Institution : Vancouver General Hospital

Department : Anaesthesia

Contact

Name : Dr A N Other

Telephone Number : (604) 875-4111

Email : DrANOther@gmail.com

Indication for Airway Management : Elective

Laparotomy

Pre-OP Physical Characteristics

Mallampati Score : IV

Thyromental Distance $<6 \mathrm{~cm}$ : Yes

Mouth Opening $<3$ Fingerbreadths? : Yes

Large Tongue? : No

Neck Mobility Reduced? : No

Radiation/Surgery to Head or Neck? : No

Congenital Abnormality? : No

Increased Aspiration Risk? : No

Raised BMI? : Yes

Bag Mask Ventilation : Two Person Technique
Sequence of events

Attempt 1

Device Used : Macintosh Laryngoscope Blade \#4 Comments : Poor view. Cormack and Lehane Grade IV Successful? : Unsuccessful

Attempt 2

Device Used : McCoy Laryngoscope

Comments : Persistent poor view $\mathrm{C} / \mathrm{L}$ grade III Successful? : Unsuccessful

Attempt 3

Device Used : Intubating LMA with endothracheal tube place fibrescopically Comments : Easy oxygenation and ventilation with LMA. Tube inserted with fibrescope

Successful? : Success

Further Comments : consider awake fibrescope

EXPORT
It is important to note that the difficultintubationapp.com does not store patient information, thus decreasing the risk of a third party obtaining personal health information. To ensure that there were no unforeseen data protection issues, we sought the advice of the VCH Information Privacy Office, who responded that they do not have "any specific privacy concerns with the application of the difficult intubation app with respect to its general application." We remind users to observe best practices regarding the appropriate storage and safekeeping of medical information. Specifically we recommend that patients add password protection access to their mobile devices.

With this simple piece of technology, available free of charge, we hope to improve transfer of this important information.

Funding Departmental funding only.
Editorial responsibility This submission was handled by Dr. Gregory L. Bryson, Deputy Editor-in-Chief, Canadian Journal of Anesthesia.

\section{References}

1. Frerk C, Mitchell VS, McNarry AF, et al.; Difficult Airway Society intubation guidelines working group. Difficult Airway Society 2015 guidelines for management of unanticipated difficult intubation in adults. Br J Anaesth 2015; 115: 827-48.

2. Apfelbaum JL, Hagberg CA, Caplan RA, et al. Practice Guidelines for Management of the Difficult Airway: an updated report by the American Society of Anesthesiologists Task Force on Management of the Difficult Airway. Anesthesiology 2013; 118: 251-70.

3. Trentman TL, Frasco PE, Milde $L N$. Utility of letters sent to patients after difficult airway management. J Clin Anaesth 2004; 16: 257-61.

Conflicts of interest None declared. 